\title{
Transplantation of artificial gelatin-co-bletillastriata gelatin/Salvia miltiorrhiza Corium promotes dermal repair in rats
}

\author{
Ge-Liang Hu, Yong-Gang Ma and Ya-Ming $\mathrm{Li}^{*}$ \\ Department of Orthopaedics, Renmin Hospital of Wuhan University, Wuhan 430060, China
}

*For correspondence: Email: yangm_li516@126.com; Tel/Fax: +86-27-88041911

Received: 25 November 2015

Revised accepted: 15 March 2016

\begin{abstract}
Purpose: To evaluate the growth of the composite corium (constructed with fibroblast cells and gelatinco-Bletillastriata gelatin/Salvia miltiorrhiza materials) on rats.

Methods: The composite artificial corium was constructed by culturing fibroblast cells in gelatin-coBletillastriata gelatin/Salvia miltiorrhiza materials. Full-thickness area of skin was excised from the mice and subsequently, the composite corium was transplanted on the wound. Thereafter, the growth difference of the composite artificial corium and natural corium were compared. In addition, real-time fluorogenic reverse transcription polymerase chain reaction (qRT-PCR) and western blot were performed to determine vascular endothelial growth factor (VEGF) expression at gene and protein levels.

Results: The composite artificial corium showed significant repair promoting effect on the skin, and the structure of the repaired skin was similar to that of natural corium. Interestingly, PCR and western blot results showed that the expressions of VEGF were higher in composite artificial corium than in natural corium on days 3 and 7 post-transplantation.

Conclusion: The composite artificial corium has some clinical prospects for use in the treatment of wounds on large areas of skin.
\end{abstract}

Keywords: Tissue engineering, Corium, Gelatin-co-Bletillastriata, Gelatin, Salvia miltiorrhiza, Vascularization, Vascular endothelial growth factor, VEGF

Tropical Journal of Pharmaceutical Research is indexed by Science Citation Index (SciSearch), Scopus, International Pharmaceutical Abstract, Chemical Abstracts, Embase, Index Copernicus, EBSCO, African Index Medicus, JournalSeek, Journal Citation Reports/Science Edition, Directory of Open Access Journals (DOAJ), African Journal Online, Bioline International, Open-J-Gate and Pharmacy Abstracts

\section{INTRODUCTION}

Skin, the largest organ of human being, plays a vital role as a barrier against infection, hurt and loss of water and electrolytes, etc. [1]. In recent years, large areas of skin and soft tissue defects have been increasingly occurring in trauma and burns, and treatment of large areas of skin defects have become a significant problem clinic $[2,3]$. Currently, the main strategy for treating skin defect is autologous skin grafts, and however the autologous skin is limited, especially for children [3]. Thus, it is important and necessary to find more ways to treat large areas of skin defects.

Recently, tissue engineering advancements have improved the understanding of wound healing and strategy for skin repair and regeneration [35]. The tissue engineered dermal equivalent could be constructed with fibroblasts and biocompatible scaffold [5]. Previous investigations have reported that gelatin and bletilla striata gelatin are good film-forming 
materials, which could be made into scaffold. Salvia miltiorrhiza can promote tissue repair and growth of wound granulation tissue via promoting the expression of endogenous epidermal growth factor (EGF), fibroblast growth factor (bFGF), vascular endothelial growth factor (VEGF), transforming growth factor- $\beta 1$ (TGF- $\beta 1$ ), and insulin-like growth factor 1 (IGF-1) [6]. In addition, the essence of granulation tissues is capillaries and fibroblasts, and therefore the Salvia miltiorrhiza has a good ability to promote angiogenesis.

In this study, by using freeze-drying the porous scaffold was prepared with raw materials of gelatin, bletilla striata gelatin and Salvia miltiorrhiza. Then, the artificial corium was constructed by adding fibroblasts into the prepared porous scaffold. Subsequently, in order to explore the feasibility of the composed corium in the clinic for treating large area of skin defect, the vascularized effects of dermal grafts were studied.

\section{EXPERIMENTAL}

\section{Materials and chemicals}

Gelatin was purchased from the Chinese Medicine Group Chemical Reagent Co., Ltd. (Shanghai, China); Bletilla striata gelatin and Salvia miltiorrhiza extracts were obtained from the Pharmaceutical laboratory of Hubei University of TCM (Wuhan, China); the composite corium was constructed by culturing fibroblast cells in gelatin-co-Bletilla striata gelatin/Salvia miltiorrhiza materials. Trizol Reagents were purchased from Invitrogen Biotech. Co. (Shanghai, China); VEGF and $\beta$ actin antibodies were purchased from Santa Cruz Biotechnology (Shanghai, China). All other chemicals used in our study were analytical reagent grade.

\section{Animals and grouping}

Male SD rats $(220-260 \mathrm{~g})$ were purchased from the Animal center of Medical College of Wuhan University (Wuhan, China). A total of 45 SD rats were randomly divided into three groups $(n=15)$, including two experimental groups (group 1 was natural corium and group 2 was artificial corium) and a control group (group 3). All animal experiments in the study were in accordance with the National Institute of Health Guide for the Care and Use of Laboratory Animals, and all the experimental protocols were approved by the Animal Care and Use Committee of the Renmin
Hospital of Wuhan University (approval ref no. 20140207\#).

\section{Animal studies}

After intraperitoneal injection (ip) of sodium pentobarbital $(40 \mathrm{mg} / \mathrm{kg})$, the rats were anesthetized and fixed. Routine disinfection and skin preparation was performed and then a $3 \times 3$ $\mathrm{cm}$ section of skin was dissected from the back of each animal and placed in saline. In the natural corium group, the skin graft was then covered and stitched in situ; in the artificial corium group, the subcutaneous tissue, including the deep fascia, were completely stripped and a $2 \times 2 \times 0.2 \mathrm{~cm}$ artificial dermis graft was placed on the wound prior to an autologous skin graft, which was then covered and stitched; in the control group, the subcutaneous tissue, including the deep fascia, were completely stripped, but only an autologous skin graft was placed on the wound, which was then covered and stitched. Sample collection was made at $3,7,14,28$ and 56 day after surgery, whereby three rats were sacrificed in each group and wound healing was observed. In the control and natural corium group, full-thickness skin tissues at the surgical sites were harvested; in the artificial corium group, full-thickness skin tissue and the artificial dermis implanted at the wound site were collected. The samples were subjected to hematoxylin and eosin (HE) staining. The expression of VEGF gene was detected by RTPCR in samples collected from days 3 and 7 , and the samples collected on day 7 were also used to detect the expression of the VEGF4 protein.

\section{HE staining assay}

The tissue samples were fixed with freshly prepared $4 \%$ formaldehyde in phosphatebuffered saline (PBS) at room temperature for 4 $\mathrm{h}$ and were then washed in PBS three times for $60 \mathrm{~min}$. Then, alcohol gradient dehydration was performed, subsequently embedded with paraffin. Then, tissue sections ( $5 \mu \mathrm{m}$ thick) were prepared with H\&E straining, according to the reported methods [7]. The histopathological changes were observed using a microscope (Olympus, Japan).

\section{Real-time fluorogenic PCR assay}

Tissues were collected, and total RNA was extracted using Trizol reagent. Total RNA was used for CDNA synthesis of VEGF and $\beta$-actin by reverse transcription using quantitative real-time PCR. All mRNA primers were designed by Primer Premier 5.0 and shown in Table 1. 
Reverse transcription was carried out according to instruction of the TOYOBO quantitative realtime RT-PCR reaction kits (Osaka, Japan).

\section{Western blot analysis}

VEGF protein expression was detected using western blot assay. Briefly, the tissues samples were collected, and total proteins were extracted. Then, protein concentration was determined using a BCA regent kit (Beyotime Biotech, Haimen, China). Then, $30 \mu \mathrm{g}$ proteins were loaded onto sodium dodecyl sulfatepolyacrylamide gel for electrophoresis (SDSPAGE) and blotted onto poly-vinylidene difluoride membrane (PVDF). Subsequently, VEGF and $\beta$ actin primary antibodies were used to determine protein expression at $4{ }^{\circ} \mathrm{C}$ for $12 \mathrm{~h}$, followed by the secondary antibodies (HPR, Beyotime Biotech, Haimen, China). Finally, the immuno reactive bands were visualized with ECLdetecting reagent (Bio-rad, USA).

\section{Statistical analysis}

All data were expressed as the mean \pm standard deviation (SD) and analyzed with one-way analysis of variance (ANOVA) with statistical software SPSS 13.0. $P<0.05$ was considered statistically significant.

\section{RESULTS}

\section{Wound healing}

As shown in Figure 1, wound healing was consistent with the conventional healing process in the natural corium group. At 7 days after transplantation, there was no visible abscess, exudation or other abnormal reaction (Fig 1B). At 14 days after transplantation, the edge of the wound became blurred and hair growth was observed (Fig 1C). At 28 days after transplantation, the surgical site appeared similar to normal tissue (Fig 1D). The same as that of rats in the natural corium group, there was no abscess, exudation or other abnormal reaction after 7 days transplantation in the artificial corium group. After 14 days of the transplantation, the grafts appeared normal with the exception of one rat, where skin graft necrosis was observed.
Notably, the edge of the wound appeared blurred and wound healing appeared to have proceeded adequately (Figure 1E). At 28 days after transplantation, wound contraction occurred, and a small amount of hair growth was observed. After 56 days, the grafts were still functional, and a small piece of graft was observed to have ischemic necrosis and was subsequently replaced by the surrounding skin. Compared to the control group, the color of the graft in the artificial corium group was light and the regrowing hair was shorter.

In the control group, significant dry shrinkage of the skin graft had occurred at 7 days after transplantation, and the wound edges of the remaining seven rats were significantly dark and gloomy. After 14 days of transplantation, grafts became dark, and at 28 days after transplantation, only four rats had functional skin grafts, and necrosis was also observed.

\section{Tissue growth}

Seven days after surgery, HE staining in the artificial corium group showed that inflammatory cells had infiltrated the artificial dermis, and new capillaries were observed and many fibroblasts were noted to be growing on the scaffold. Fourteen days after surgery, the porous scaffold was completely surrounded by tissue, fibroblasts were growing steadily and the new blood capillaries were confluent (Fig 2A). At day 28, the material had been completely absorbed and many new collagen fibers with irregular arrangements were in place, the neo-vasculature was more mature and wound contraction was observed (Fig 2B). At day 56, the structure of the skin was similar to normal rats, but lacked hair follicles and sweat glands, and there was a gap between the dermis and the deep tissues (Figure $2 \mathrm{C})$.

\section{VEGR expression}

RT-PCR results showed that the VEGF gene was expressed in damaged tissues and the expression of VEGF was significantly higher in the natural and artificial groups than that in control group at 3 days after transplantation. The

Table 1: Primers used for PCR

\begin{tabular}{lll}
\hline Variable & Sequence $\left(5^{\prime}-3^{\prime}\right)$ & Length \\
\hline \multirow{2}{*}{ VEGF } & TGTGAGCCTTGTTCAGAGCG & $252 \mathrm{bp}$ \\
\multirow{2}{*}{$\boldsymbol{\beta}$-actin } & GACGGTGACGATGGTGGTGT & \\
& CGTTGACATCCGTAAAGACCTC & $110 \mathrm{bp}$ \\
\hline
\end{tabular}



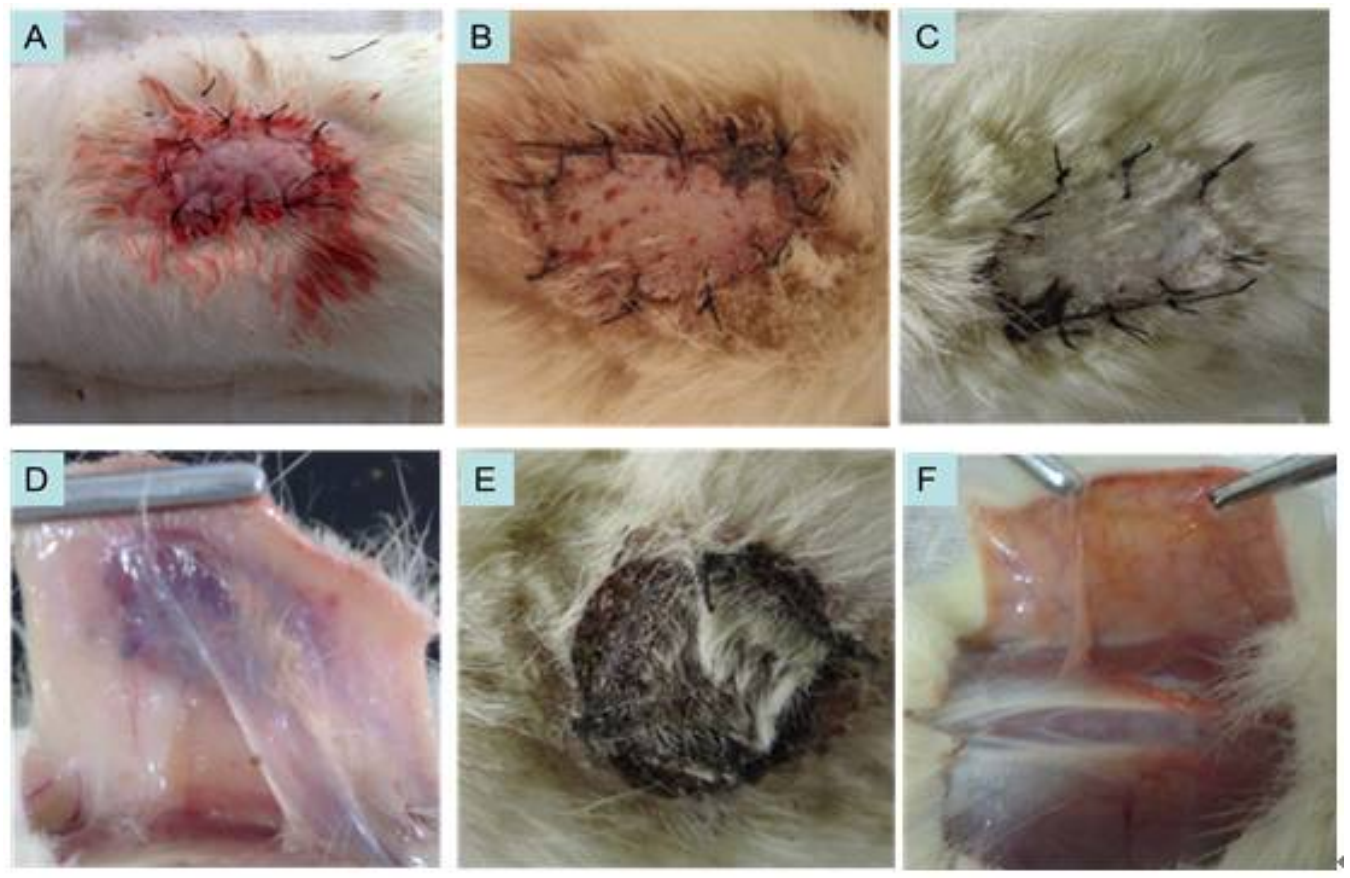

Figure 1: Results of the wound healing. A. Derma transplantation; B.7 days after transplantation in natural corium group. C. 14 days after transplantation in natural corium group; D. 28 days after transplantation in natural corium group; E. 14 days after transplantation in artificial corium group; F. 28 days after transplantation in control group
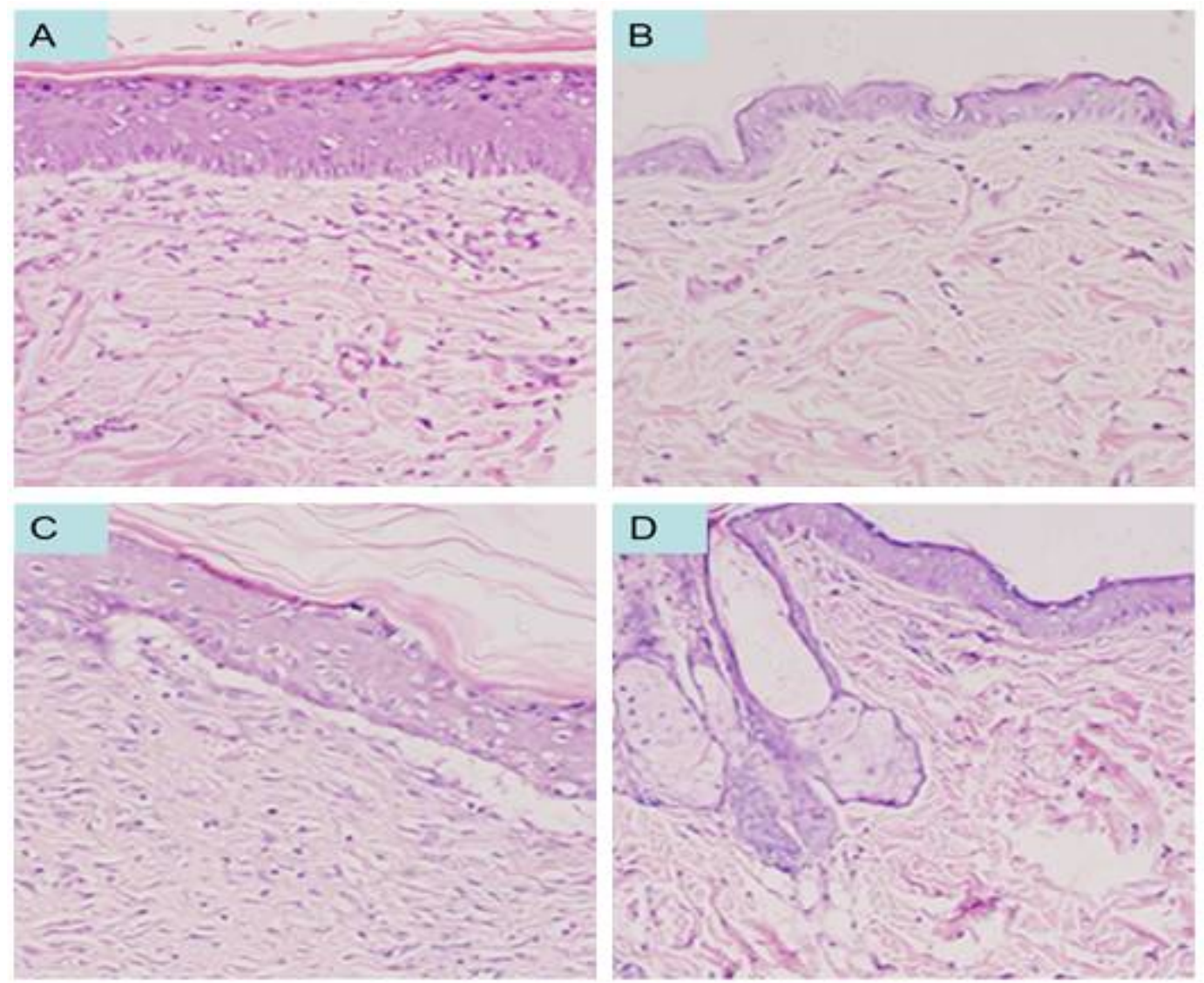

Figure 2: Results of the HE staining assay. A. At 14 days after transplantation in artificial corium group; B. At 28 days after transplantation inartificial corium group; C. At 56 days after transplantation inartificial corium group; D. At 56 days after transplantation in control group 


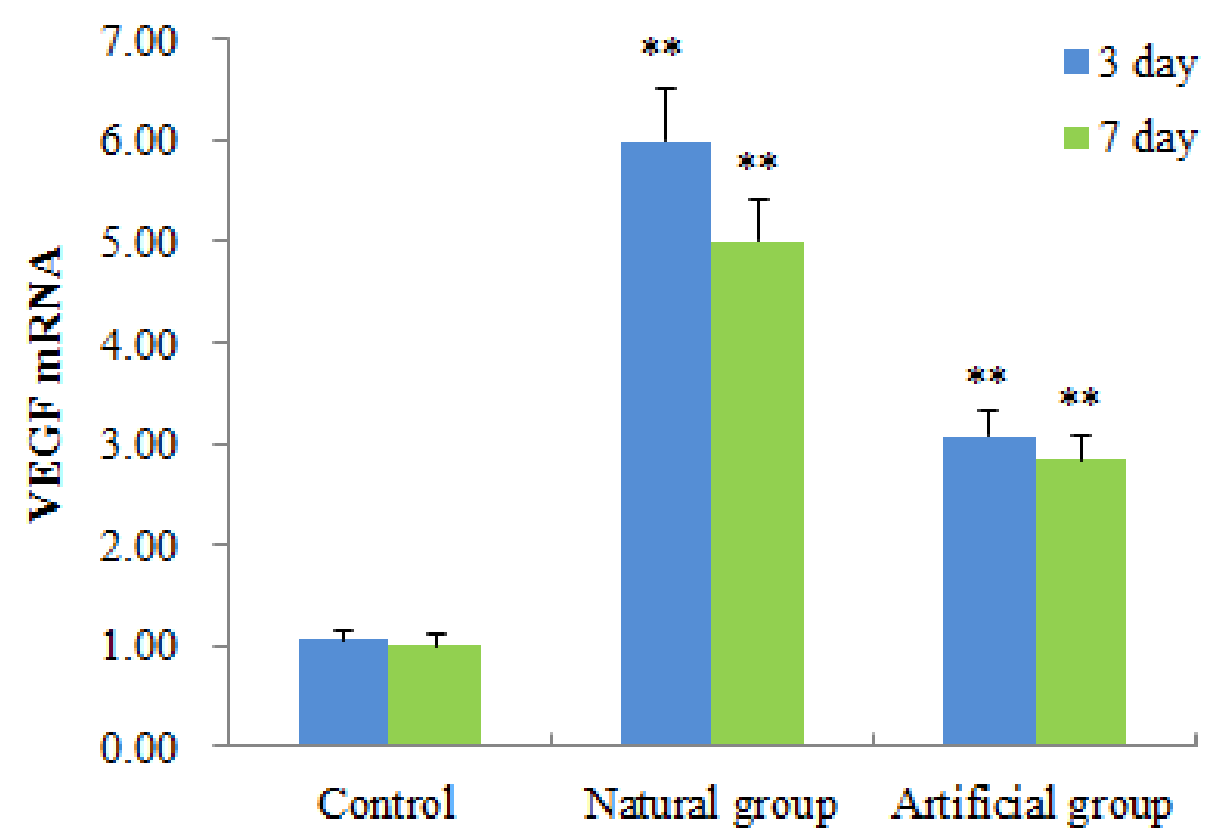

Figure 3: VEGR expressions
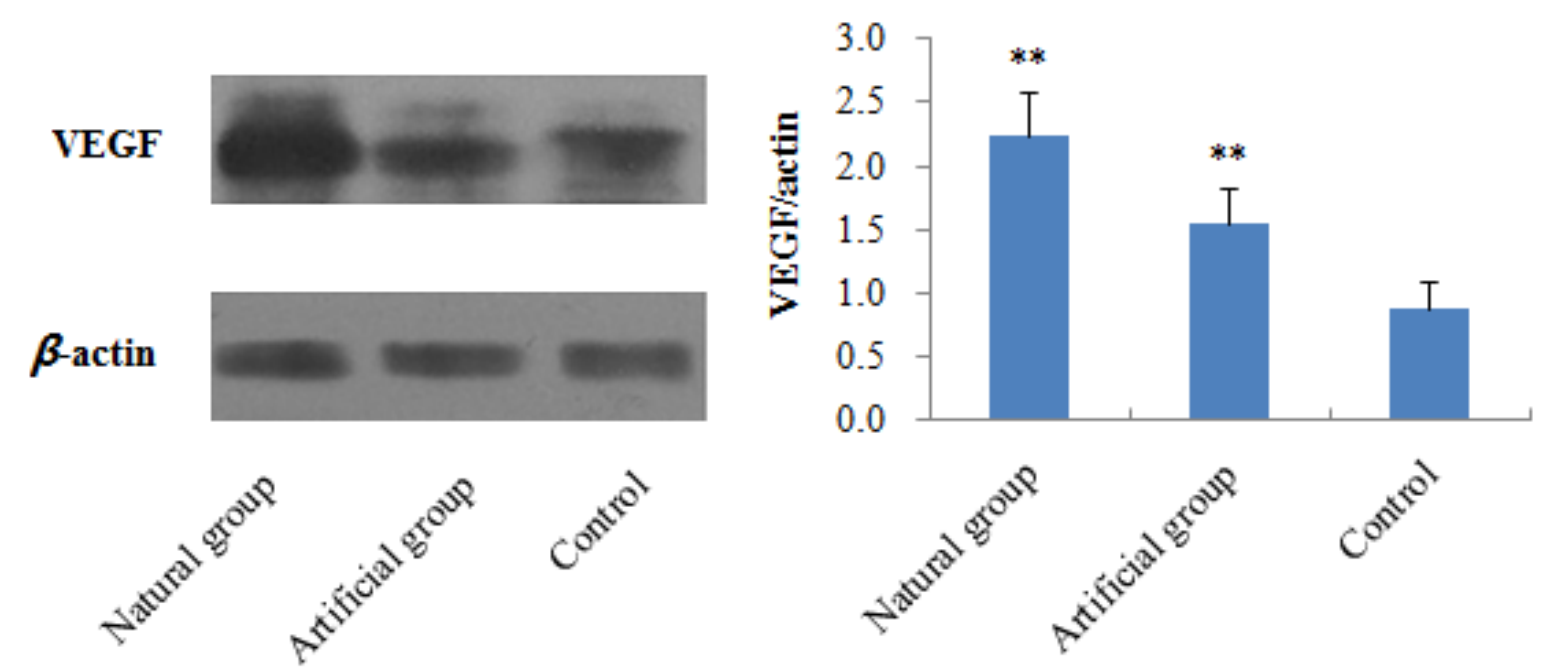

Figure 4: VEGF expression by western blot assay. To normalize for protein loading, VEGF expression level was expressed as a relative value to that of $\beta$-actin

expression level decreased on the 7th day compared to the 3rd day (Figure 3). In general, the expressions of VEGF in rats that received the artificial dermis were lower than that of rats in the natural dermis group.

As can be seen from Figure 4, our western blot assay results showed that VEGF expressions in the two experimental groups were significant higher than that in the control group $(p<0.01)$. However, our results also indicated that the expression of the VEGF protein in the artificial dermis group was lower than in the natural dermis group.

\section{DISCUSSION}

Skin tissue engineering uses modern biology and engineering principles to construct tissue substitutes for damaged tissues [8]. Artificial skin has been successfully used to repair large areas of defective skin resulting from trauma or burns $[9,10]$. However, artificial skin suffers from a number of disadvantages. For example, since only a layer of the epithelia is covered on the wound, the surviving epithelial tissues, with poor elasticity, can easily break and contract. Previously, histology has shown that the epithelial basement membrane contains incomplete collagen fibers and anchors [11].

Trop J Pharm Res, April 2016; 15(4): 739 
Therefore, research has focused on better dermal substitutes [12].

In this regard, researchers found that the addition of $\beta$-FGF to the artificial dermis could accelerate tissue regeneration [13].

Meanwhile, Wilcke et al found that incorporation of VEGF and $\beta$-FGF into fibrin matrix materials could result in the construction of dermal substitutes, which could accelerate the formation of new blood vessels and improve graft survival when replanted into the full-thickness skin wounds of nude mice [14]. Furthermore, Pienimaki et al [15] found that EGF could significantly accelerate wound healing and promote keratinocytes to produce hyaluronan and Cuono et al [16] constructed a deepidermalized dermis (DED) with cadaver skin, which retained the scaffold and full basement membrane complex without immunogenicity, and the epidermal cells could differentiate into a multilayered epidermis on the DED. Hansbrough et al [17] cultured fibroblasts on poly glycolic acid (PGA) fibers in vitro for 2-3 weeks and then used it to repair a skin wound. They found that new blood vessels formed, fibroblasts proliferated and a collagen matrix formed.

In order to accelerate vascular formation and wound healing, many studies have been performed in which angiogenic drugs and fibroblast culture have been used and shown to be applicable techniques. Coulommb et al [18] constructed a dermal substitute with fibroblasts and collagen gel, and then transplanted the dermis onto rats. The results showed that the fibroblasts grew and proliferated on the wound. Meanwhile, Yamada et al [19] treated 26 wound sites with a fibroblast-cultured collagen sponge and the results showed that wound healing was significantly accelerated.

In our study, fibroblasts were cultured on a gelatin-co-Bletilla striata gelatin/Salvia miltiorrhiza composite to construct an artificial dermis, which was then transplanted onto the dermis of rats. The results showed that the artificial dermis integrated into the skin graft after 7 days, and there was obvious dermis-like tissue formation after 14 days. Furthermore, the artificial materials were absorbed and the artificial skin began to replace the defective skin after 28 days. On day 56, the resulting dermal structure appeared similar to normal skin. VEGF is the most important factor in wound healing $[20,21]$ and is expressed approximately 1 day after skin injury, reaching a peak after 3-4 days. Its levels finally return to normal after $10-14$ days [22]. Therefore, the expressions of the VEGF gene and protein were detected to assess wound healing. The results showed that the expression of VEGF was significantly high in the artificial dermis and natural dermis groups after 3 and 7 days.

\section{CONCLUSION}

The developed gelatin-co-Bletilla striata gelatin/Salvia miltiorrhiza composite may be applied as a dermal substitute to promote vascularization of damaged skin tissue. However, the composite still needs to be further investigated to confirm its effects on the repair of epidermal tissue.

\section{REFERENCES}

1. Butler CE, Orgill DP. Simultaneous in vivo regeneration of neodermis, epidermis, and basement membrane. Adv Biochem Eng Biotechnol 2005; 94: 23-41.

2. Hrabchak C, Flynn L, Woodhouse KA. Biological skin substitutes for wound cover and closure. Expert Rev Med Dev 2006; 3: 373-385.

3. Zhao S, Li L, Wang $H$, Zhang $Y$, Cheng $X$, Zhou $N$, Rahaman MN, Liu Z, Huang W, Zhang C. Wound dressings composed of copper-doped borate bioactive glass microfibers stimulate angiogenesis and heal fullthickness skin defects in a rodent model. Biomaterials 2015; 53: 379-391.

4. Castangia I, Nácher $A$, Caddeo $C$, Valenti $D$, Fadda AM, Díez-Sales O, Ruiz-Saurí A, Manconi M. Fabrication of quercetin and curcumin bionanovesicles for the prevention and rapid regeneration of full-thickness skin defects on mice. Acta Biomater 2014; 10: 1292-1300.

5. Balasubramani M, Kumar TR, Babu M. Skin substitutes: a review. Burns 2001; 27: 534e44.

6. Wang $Y$, Wang S, Li Y, Jiang J, Zhou C, Li C, Li D, Lu L, Liu $P$, Huang $M$. Therapeutic effect of Cryptotanshinone on collagen-induced arthritis in rats via inhibiting nuclear factor kappa B signaling pathway. Transl Res 2015; 165: 704-716.

7. Peng W, Hu C, Shu Z, Han T, Qin L, Zheng C. Antitumor activity of tatariside $F$ isolated from roots of Fagopyrum tataricum (L.) Gaertn against H22 hepatocellular carcinoma via up-regulation of p53. Phytomed 2015; 22 : 730-736.

8. Sun JZ, Han CM. The advance of tissue engineering skin. Int J Surg 2006; 33: 389-391.

9. Langer R, Vacanti JP. Tissue engineering. Science 1993; 260: 920.

10. Hansbrough JF, Mozingo DW, Kealey GP, Davis M, Gidner A, Gentzkow GD. Clinical trials of a biosynthetic temporary skin replacement, dermagraft-transitional covering, compared with cryopreserved human cadaver skin for temporary coverage of excised burn wounds. J Burn Care Rehabil 1997; 18: 43-51. 
11. Woodley D, Peterson HD, Herzog SR. Burn wound resurfaced by cultured epidermal autografts show abnormal reconstitution of anchoring fibrils. JAMA 259: 2566-2571, 1988.

12. Sibbald. Apligraft ${ }^{T M}$ living skin equivalent for healing venous and chronic wounds. J Cuta Med Surg 1998; 3 : 24-28.

13. Kawai K, Suzuki S, Tabata Y. Accelerated tissue regeneration through incorporation of basic fibroblast growth factor-impregnated gelatin microspheres into artificial dermis. Biomate 2000; 21: 489-449.

14. Wilcke I, Lohmeyer JA, Liu S. VEGF and $\beta$-FGF protein based therapy in a slow release system to improve angiogenesis in a bioartificial dermal substitute in vitro and in vivo. Langenbecks Arch Surg 2007; 392: 305314.

15. Pienimaki JP, Rilla K, Fulop C. Epidermal growth factor activates hyaluronan synthase in epidermal keratinocytes and increases pericellular and intracellular hyaluronan. J Bio Chem 2001; 276: 20428-2043.

16. Cuono $C B$, Langdon $R$, Birchall $N$. Composite autologous-allogeneic skin replacement: development and clinical application. Plast Reconstr Surg 1987; 80: 626-637.
17. Hansbrough JF, Mozingo DW, Kealey GP. Clinical trials of a biosynthetic skin replacement, DermagraftTransitional Covering, compared with cryopreserved human cadaver skin for temporary coverage of excised burn wounds. J Burn Care Rehabil 1997; 18: 43-51.

18. Coulommb B, Lebreton $C$, Dubertret $L$. Influence of human dermal fibroblasts on epidermalization. $J$ Invest Dermatol 1989; 92: 122-125.

19. Yamada N, Uchinuma E, Kuroyanagi Y. Clinical evaluation of anallogeneic cultured dermal substitute composed of fibroblasts within a spongy collagen matrix. Scand J Reconstr Hand Surg 1999; 33: 147-154.

20. Harmey JH, Dimitriadis E, Kay E. Regulation of macrophage production of vascular endothelial growth factor (VEGF) by hypoxia and transforming growth factor -1. Ann Sur Oncol 1998; 5: 27.

21. Soker $S$. Neuropilin in the midst of cellmigration and retraction. Int J Biochem Cell Biol 2001; 33: 433-437.

22. Corral CJ, Siddiqui $A$, Wu L. Vascular endothelial growth factor is more important than basic fibroblastic growth factor during ischemic wound healing. Arch Surg 1999; 134: 200-205. 